\title{
VARIATIONS IN ACCESSIBILITY OF ROUND WINDOW VIA POSTERIOR TYMPANOTOMY APPROACH IN COCHLEAR IMPLANT SURGERY
}

\author{
Uzair Mushahid, Sayed Nusrat Raza*, Muhammad Ali, Shoaib Ahmed, Abdul Hakim, Shakeel Ahmed \\ Combined Military Hospital/National University of Medical Sciences (NUMS) Rawalpindi Pakistan, *Army Medical College/National University of Medical \\ Sciences (NUMS) Rawalpindi Pakistan
}

\begin{abstract}
Objective: To apply the St Thomas' Hospital (STH) classification of round window type, in a Pakistani pediatric population undergoing cochlear implantation, and rate the inter observer variability of applying this classification.

Study Design: Cross sectional study.

Place and Duration of Study: Combined Military Hospital Rawalpindi, from Apr 2019 to Dec 2020.

Methodology: Patients were examined per-operatively by a panel of four surgeons after "optimal" posterior tympanotomy for round window variations, as per STH classification of approachability of RWM. The observations of the four surgeons were recorded and interobserver variation was assessed and analyzed.

Results: A total of 100 patients were operated, 45 females and 55 males. Mean age was 3.8 years. There was minimal inter observer variability with regards to round window type and extent of "optimal" posterior tympanotomy. Three patients had type I, 76 had type IIA, 15 had type IIB and 6 patients had type III. Round window insertion/membranous cochleostomy was possible in 70 patients, whereas the rest require extended round window approach or bony cochleostomy.

Conclusion: The STH classification is a useful predictor of route of CI electrode insertion and most patients can undergo RW insertion with confidence based on minimal variation between surgeons when applying the STH classification as well as when deciding the extent of surgical exposure.
\end{abstract}

Keywords: Cochlear implant (CI), Deafness.

How to Cite This Article: Mushahid U, Raza SN, Ali M, Ahmed S, Hakim A, Ahmed S. Variations in Accessibility of Round Window via Posterior Tympanotomy Approach in Cochlear Implant Surgery. Pak Armed Forces Med J 2021; 71 (Suppl-3): S452-456. Doi: https://doi.org/10.51253/pafmj.v1i1.6039

This is an Open Access article distributed under the terms of the Creative Commons Attribution License (https://creativecommons.org/licenses/by-nc/4.0/), which permits unrestricted use, distribution, and reproduction in any medium, provided the original work is properly cited.

\section{INTRODUCTION}

Congenital hearing loss in the Pakistani population may be as high as 13 per $1000 .{ }^{1}$ Cochlear implant (CI) is an FDA approved device for the treatment of congenital profound sensorineural hearing loss (SNHL). Implantation at early age result in better auditory comprehension outcomes. ${ }^{2}$ The surgical approach for the cochlear implant electrode insertion should aim to minimize intracochlear trauma and ensure entry into the scala tympani. ${ }^{3}$ The two most popular techniques for electrode insertion are round window membrane (RWM) insertion and bony cochleostomy; the former is considered to be less traumatic and more likely to preserve residual hearing. ${ }^{4}$

There might be anatomic variations in the RW area that may render it a challenging approach. ${ }^{5}$ The RWM is located in a small niche called fossula fenestrae rotunda and often hidden behind overhanging bony ridges from superior, posterior and anterior, which regularly limits the visibility of the RWM during surgery. ${ }^{6}$ The surgical technique consists of drilling a space for the receiver/stimulator then performing a cortical mastoidectomy, followed by a posterior tym-

Correspondence: Dr Uzair Mushahid, Department of ENT, Combined Military Hospital, Rawalpindi Pakistan panotomy. ${ }^{7}$ Posterior tympanotomy is an approach to access the middle ear by drilling a space between the facial nerve and chorda tympani. This is required to access the round window and promontory. ${ }^{8}$ The insertion of CI electrode via RWM is considered more difficult in children, compared to adults. ${ }^{9,10}$ Cochleostomy for type IIB, and bony cochleostomy for type III. ${ }^{11}$

Currently there are no practical guidelines to help surgeons choose the surgical approach for CI and most decisions are made peropertively based on the surgeon's judgment and preference. Although the STH classification can be used as a reference for operating surgeons to decide the most appropriate technique, inter observer variability may lead to differences in surgical approach. In view of these gaps in practice, the purpose of our study was to evaluate the utility of STH classification in predicting the appropriate route of CI electrode insertion in a Pakistani pediatric population, and whether there is any significant inter observer variability in applying the STH classification and assessing the extent of surgical exposure.

\section{METHODOLOGY}

A cross sectional study was conducted on consecutive pediatric patients who had received clearence from Multi Disciplinary Team (MDT; consisting of 
Otologists, pediatricians, pshychologists, audiologists and speech therapists) to undergo cochlear implantation. The study was carried out at Combined Military Hospital Rawalpindi, from April 2019 and December 2020, which is a large tertiary care hospital located in the north of the country.

Ethical approval was obtained from institutional review board prior to commencing study, and informed consent was obtained from patients' parents.

Inclusion criteria: Pediatric patients with congenital sensorineural hearing loss, documented with Auditory brainstem response (ABR) audiometry, normal structure of external, middle and inner ear and normal CNVII and CNVIII on CT scan and MRI, physically fit for general anesthesia and those in whom full insertion of the electrode was possible.

Exclusion criteria: Patients above the age of 5 years, with structural anomalies of the ear or Cranial nerves VII/VIII, with hearing serviceable by conventional hearing aids, those in whom full electrode insertion was not possible or in whom surgery was abandoned /postponed due to anesthetic/surgical complications, those undergoing revision surgeries and those expected to have low compliance to speech therapy or follow up after surgery and those who had not received approval for cochlear implant by MDT.

The St Thomas Hospital classification (figure-1) is based on multiple per op findings of adult and pediatric cochlear implants. It grades the visibility of the RWM into four types. After an "optimal" posterior tympanotomy, the round window niche bony overhang is drilled without breaching RWM. An "optimal" posterior tympanotomy is defined as an operating surgeon's best surgical effort to achieve the widest posterior tympanotomy whilst preserving the integrity of the facial nerve, chorda tympani, posterior canal wall and bony annulus, so being able to dissect out the best exposure possible of the RWM by removing the round

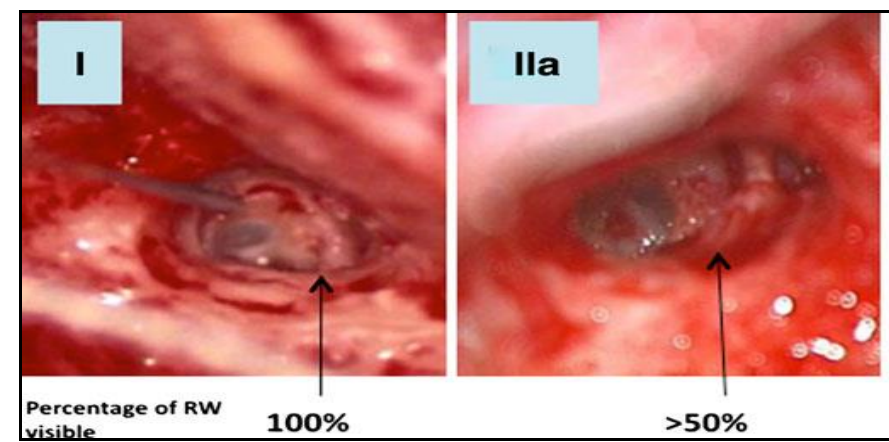

$>50 \%$ window niche bony overhang. ${ }^{11}$

Four surgeons separately classified the RWM type at time of posterior tympanotomy and also commented on whether the posterior tympanotomy was "optimal" in their opinion. Verbal response were collected from operating surgeons and recorded by the researcher at time of surgery. If of all 4 surgeons were in agreement, it was considered as "full consensus", if 3 out of 4 surgeon were in agreement it was considered as "majority consensus", and if 2 out of 4 surgeons agreed, it was considered as "half consensus". In cases of "half consensus" the opinion of the senior most surgeon was considered final. We defined $75-100 \%$ "full consensus" as minimum inter observer variability, 50-74\% "full consensus" as moderate inter observer variability and less than $50 \%$ "full consensus" as substantial inter observer variability.

Based on the round window type, the electrode insertion technique was finalised: round window insertion (membranous cochleostomy), extended RW approach or bony cochleostomy. The preferred method was membranous cochleostomy, if that was not possible then extended RW approach, and if that was not possible, or if no part of the RW was visible, then bony cochleostomy was performed. The cochlear implant used in the study was MED-ELTM synchrony (Figure2). The data was analyzed with SPSS version 25 .
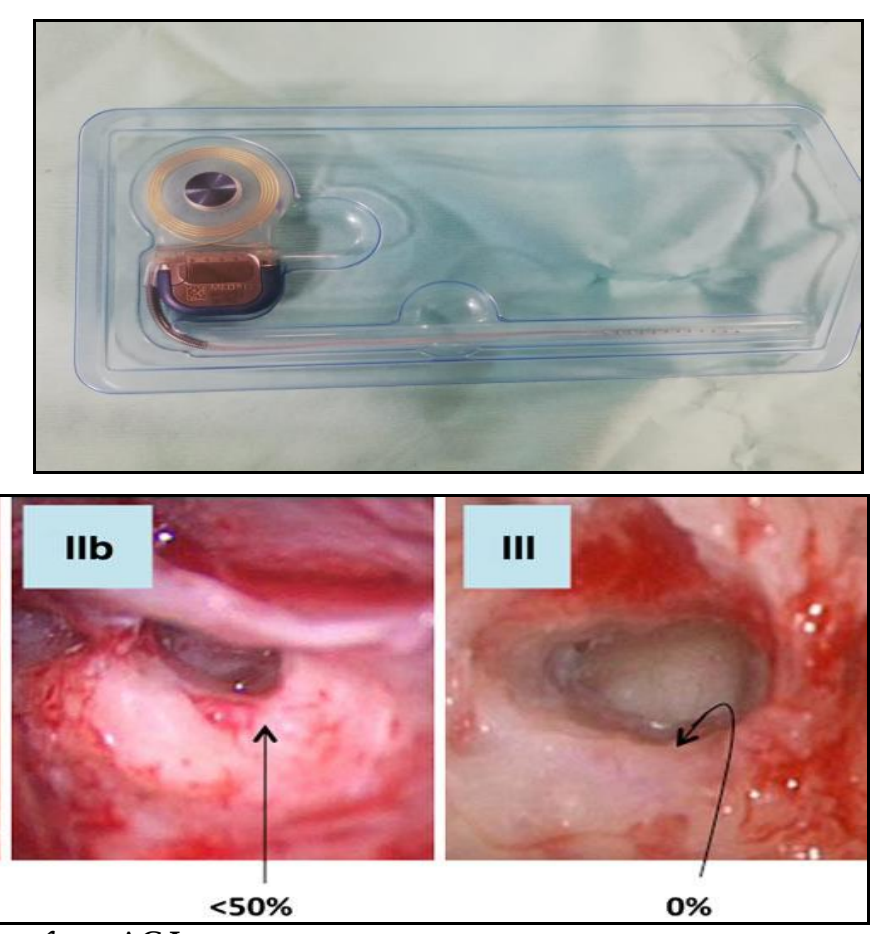

visible

$<50 \%$

$\mathbf{0} \%$

Figure-1: STH Round window classification, used with permission from AC Leong. 
Figure-2: Cochlear implant: MED El synchrony.

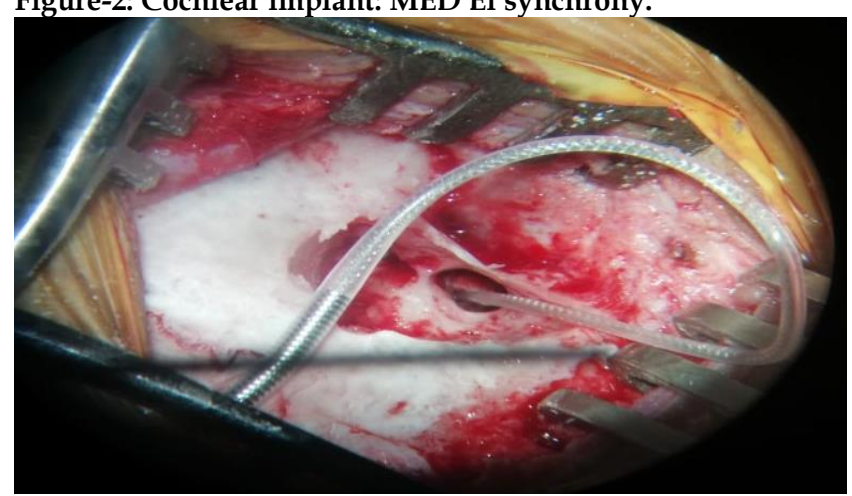

Figure-3: Electrode in round window, through posterior tympanotomy.

\section{RESULTS}

One hundred consecutive pediatric patients who met the inclusion criteria and underwent cochlear implant surgery were included in the study. All underwent $\mathrm{CI}$ in right ear. Out of 45 patients were females and 55 were males. Mean age of the patients was 3.8 years.

The results of the Round window type and consensus on "optimal" posterior tympanotomy are summarized in Table-I \& II respectively.

\begin{tabular}{l|c|c|c} 
Table-I: Round windows types \\
\hline $\begin{array}{l}\text { Round } \\
\text { Window } \\
\text { Type }\end{array}$ & $\begin{array}{c}\text { No. of } \\
\text { Patients } \\
(\mathbf{n = 1 0 0 )}\end{array}$ & \multicolumn{2}{|c}{$\begin{array}{c}\text { Consensus on } \\
\text { Round Window Type }\end{array}$} \\
\hline I & 3 & Full Consensus & $100 \%(3 / 3)$ \\
\hline \multirow{2}{*}{ IIA } & \multirow{2}{*}{76} & Full Consensus & $80 \%(61 / 76)$ \\
\cline { 3 - 4 } & & Majority Consensus & $17 \%(13 / 76)$ \\
\cline { 3 - 4 } & \multirow{2}{*}{ IIB } & Half Consensus & $2.6 \%(2 / 76)$ \\
\hline III & 6 & Full Consensus & $87 \%(13 / 15)$ \\
\cline { 3 - 4 } & & Majority Consensus & $13 \%(2 / 15)$ \\
\hline
\end{tabular}

\begin{tabular}{l|c|c|c}
\hline \multicolumn{3}{l}{$\begin{array}{l}\text { Table-II: } \\
\text { posterior tympanotomy }\end{array}$} \\
\begin{tabular}{l|c|c} 
Round \\
Window \\
Type
\end{tabular} & $\begin{array}{c}\text { No. of } \\
\text { Patients } \\
(\mathbf{n = 1 0 0 )}\end{array}$ & \multicolumn{2}{|c}{$\begin{array}{c}\text { Consensus on "Optimal" Posterior } \\
\text { Tympanotomy }\end{array}$} \\
\hline I & 3 & Full consensus & $100 \%(3 / 3)$ \\
\hline \multirow{2}{*}{ IIA } & \multirow{2}{*}{76} & Full consensus & $77.6 \%(59 / 76)$ \\
\cline { 3 - 4 } & & Majority consensus & $18.4 \%(14 / 76)$ \\
\cline { 3 - 4 } & \multirow{2}{*}{ IIB } & Half consensus & $4 \%(3 / 76)$ \\
\cline { 3 - 4 } & \multirow{2}{*}{15} & Full consensus & $60 \%(9 / 15)$ \\
\cline { 3 - 4 } & & Majority consensus & $26.6 \%(4 / 15)$ \\
\hline \multirow{2}{*}{ III } & \multirow{2}{*}{6} & Full consensus & $13.3 \%(2 / 15)$ \\
\cline { 3 - 4 } & & Majority consensus & $67 \%(4 / 6)$ \\
\hline
\end{tabular}

All patients with type 1 RWM had round window (membranous cochleostomy) insertion of electrodes. In patients with type IIA RWM, $85.5 \%$ had round window (membranous cochleostomy) insertion and 14.5\% required extended round window insertion. in patients with Type IIB RWM, 13.3\% had round window (membranous cochleostomy) insertion, $60 \%$ had extended round window insertion and $26.6 \%$ had bony cochleostomy. All patients with type III RWM required bony cochleostomy.

All electrode insertions were confirmed by post operative skull $x$-ray. Three patients developed post op temporary facial paresis, all of whom recovered completely within 4 months. There were no cases of permanent facial palsy. Four patients complained of slight bleeding from the nose, which resolved within 2-3 days. There were 2 cases of electrode displacement, discovered on post operative $X$ ray, both cases were re explored within 24 hours.

\section{DISCUSSION:}

In our study, full consensus on the type of RWM was found in 83 out of 100 patients, majority consensus in 15 patients and half consensus was present in only 2 patients. Hence there was minimum inter observer variability in defining the RW type. With regards to extent of "optimal" posterior tympanotomy, full consensus was reached in 75 out of 100, majority consensus in 20 and half consensus in 5 patients. The inter observer variability is also minimum, although it is more than it is with RWM type (75\% vs $83 \%$ ). One possible explanation for variability in extent of "optimal" posterior tympanotomy is that the operating surgeon could be erring on the side of caution and thus compromised ideal exposure for the sake of preservation of structures. In comparison, Leong et al. have also stated that Inter and intra-observer variability of the grading of RW type was minimal in their study. ${ }^{11}$ Our study adds to the evidence that STH classification is a fairly robust method to classify RWM types.

In our study, type I RWM was not as common as reported by Leong et al, (3\% vs 46\%). ${ }^{11}$ Our study had higher percentage of type IIA RW (76\% vs $47 \%)$. Similar to Leong et al, and Stuermer et al, majority of our patients had more than $50 \%$ of the round window visible (i.e Type I and Type IIA); $79 \%$ in our study, $78 \%$ in Leong et al, and $86 \%$ in Stuermer et al. ${ }^{11,12}$ This indicates that majority of the patients had favorable anatomy and that RWM insertion, via membranous cochleostomy is feasible in most cases.

Based on the RW type, the route of electrode insertion could be predicted. Although bony cochleostomy enables residual hearing preservation, however multiple studies have shown that RW approach has better residual hearing outcomes compared to bony cochleostomy. ${ }^{13,14}$ Furthermore, cochleostomy is associa- 
ted with potentially greater adverse effects such as perilymph leak, acoustic trauma and contamination with bone dust, whereas RW approach minimizes such trauma. ${ }^{15}$ Therefore, our preferred insertion approach was RW and bony cochleostomy was selected as last resort. Out of 100 patients in our study, round window insertion (membranous cochleostomy) was possible in 70 patients, most of which comprised of type I and type IIA RWM types. In comparison, Leong et al. had RW insertion in $88 \%$ of cases, comprising of both adult and pediatric patients. ${ }^{11}$

Leong et al,11 have defined an "optimal" posterior tympanotomy as an operating sur geon's best surgical effort to achieve the widest posterior tympanotomy whilst preserving the integrity of the facial nerve, chorda tympani, posterior canal wall and bony annulus, so being able to dissect out the best exposure possible of the RWM by removing the round window niche bony overhang. In our study we have followed a similar method with regards to defining extent of posterior tympanotomy, which is mainly based on operating surgeons' judgment, and this still remains subjective. ${ }^{16}$ Stuermer et al, have more precisely defined anatomical parameters of "optimal surgical exposure" and this may have contributed to achievement of higher rate of RWM visibility compared to Leong et al. In addition, Stuermer et al, have also discussed the mean dimensions of the posterior tympanotomy which allowed them adequate exposure and electrode insertion; an opening size of $4.3 \mathrm{~mm}$ in cranio-caudal orientation to $3.2 \mathrm{~mm}$ in anterior orientation. ${ }^{12}$ They do mention a caveat that the dimensions of the posterior tympanotomy do not necessarily correlate with the visibility of the round window, since other factors play a role here, such as the angle at which the individual landmarks are located. Round window type may not be the sole predictor for ease of electrode insertion. Round window has variable anatomy and the shape of the round window could vary from saddle shaped, ovoid to triangular. ${ }^{17}$

A number of studies have studied parameters in pre op imaging that might be indicative of ease of electrode insertion through RWM.16,18,19 Leong et al, mention that imaging did not help them much in predicting the RWM type (although they only had MRI done in their institute). In our study both MRI and CT scan were done pre operatively. Similar to Leong et al, imaging of normal ears was not helpful in pre operatively predicting the round window type or the ease of electrode insertion.
In present study, one unexpected complication encountered was postoperative nose bleed. This is not widely reported in the literature. ${ }^{20}$ One possible explanation for this complication is leakage of blood or blood tinged saline via the Eustachian tube into the nasopharynx and nasal cavity.

To ensure the standardization of results, our study included CIs of only one brand in all our patients, whereas in both Leong et al, and Steurmer et al, CI of different brands were used in the same study. ${ }^{11,12}$ Previous studies had included both adult and pediatric populations whereas our study only included pediatric population.

One of the limitations of the STH classification is the subjective nature of the classification, hence we suggest that to reduce the amount of inter observer variation and the subsequent decision for the surgical approach, a method similar to the one used in our study can be applied whereby a majority consensus of a team of otologists can be used to determine the most appropriate RW type and surgical approach.

\section{ACKNOWLEDGMENT}

Special thanks to Dr. Iqbal Khan (Bradford Teaching hospital/Yorkshire Cochlear implant Services, United kingdom) for overseeing and mentoring our cochlear implant program.

\section{LIMITATION OF STUDY}

Optimal posterior tympanotomy is still subjective and we had not defined it's exact anatomical boundaries or size. Long term complications and outcomes were not included in our study. Blinding and randomisation of the cases was not done.

\section{CONCLUSION}

The STH classification is a useful predictor of route of CI electrode insertion and most patients can undergo RW insertion. There is minimal variation between surgeons when applying the STH classification as well as when deciding the extent of surgical exposure. Obtaining consensus can provide better confidence to the operating surgeon and promote better decisions regarding optimum surgican technique.

\section{Conflict of Interest: None.}

\section{Authors' Contribution}

UM: Main author, SNR: Study concept, principal supervisor, MA: Patient data collection, SA: Literature review, AH: Patient follow-up, SA: Proof reading.

\section{REFERENCES}

1. Ahmed S, Sheraz S. Frequency of congenital hearing loss in neonates. J Ayub Med Coll Abbottabad 2018; 30(2): 234-236.

2. Mitchell RM, Christianson E, Ramirez R. Auditory comprehension outcomes in children who receive a cochlear implant before 12 months of age. Laryng 2020; 130(3): 776-781. 


\section{Cochlear Implant Surgery}

3. Hoskison E, Mitchell S, Coulson C. Systematic review: radiological and histological evidence of cochlear implant insertion trauma in adult patients. Cochlear Implants Int 2017; 18(4): 192-197.

4. Pau HW, Just T, Bornitz M, Lasurashvilli N, Zahnert T. Noise exposure of the inner ear during drilling a cochleostomy for cochlear implantation. Laryng 2007; 117(3): 535-540.

5. Singla A, Sahni D, Gupta AK, Loukas M, Aggarwal A. Surgical anatomy of round window and its implications for cochlear implantation. Clin Anat 2014; 27(3): 331-336.

6. Luers JC, Hüttenbrink KB, Beutner D. Surgical anatomy of the round window-implications for cochlear implantation. Clin Otolaryngol 2018; 43(2): 417-424.

7. Weinreich HM, Francis HW, Niparko JK, Chien WW. Techniques in cochlear implantation. Oper Tech Otolaryngol-Head Neck Surg 2014; 25(4): 312-320.

8. Kumar R, Singh A, Sagar P, Behera C, Kumar R. Access to round window niche via posterior tympanotomy and impact of drilling its overhangs: a cadaveric descriptive study. Indian J Otolaryngol Head Neck Surg 2018; 70(4): 510-514.

9. Lloyd SKW, Kasbekar AV, Kenway B. Developmental changes in cochlear orientation-implications for cochlear implantation. Otol Neurotol 2010; 31(6): 902-907.

10. McRackan TR, Reda FA, Rivas A. Comparison of cochlear implant relevant anatomy in children versus adults. Otol Neurotol 2013; 33(3): 328-334.

11. Leong AC, Jiang D, Agger A, Fitzgerald-O'Connor A. Evaluation of round window accessibility to cochlear implant insertion. Eur Arch Oto-Rhino-Laryngol 2013; 270(4): 1237-1242.
12. Stuermer K, Round window accessibility during cochlear implantation. Eur Arch Oto-Rhino-Laryngol 2020; 278(2): 363-370.

13. Bae SC, Shin YR, Chun YM. Cochlear implant surgery through round window approach is always possible. Ann Otol Rhinol Laryngol 2019; 128(suppl-6): 38S-44S.

14. Snels C, Inthout J, Mylanus E, Huinck W, Dhooge I. Hearing preservation in cochlear implant surgery: a meta-analysis. Otol Neurotol 2019; 40(2): 145-153.

15. Adunka O, Unkelbach MH, Mack M, Hambek M, Gstoettner W, Kiefer J. Cochlear implantation via the round window membrane minimizes trauma to cochlear structures: A histologically controlled insertion study. Acta Otolaryngol 2004; 124(7): 807-812.

16. Park E, Amoodi H, Kuthubutheen J, Chen JM. Predictors of round window accessibility for adult coch-lear implantation based on pre-operative CT scan: a prospective observational study. J Otolaryngol-Head Neck Surg 2015; 44(1): 1-7.

17. Jain S, Gaurkar S, Deshmukh PT. Applied anatomy of round window and adjacent structures of tympanum related to cochlear implantation. Braz J Otorhinolaryngol 2019; 85(4): 435-446.

18. Canzi P, Avato I, Manfrin M. Anatomic variations of the round window niche: radiological study and related endoscopic anatomy. Surg Radiol Anat 2019; 2(1): 1-4.

19. Chen J, Wu Y, Shi J. Predictors of round window membrane visibility in pediatric cochlear implant surgery using temporal bone HRCT: a retrospective study. Int J Pediatr Otorhinolaryngol 2019; 121(2): 150-153.

20. Halwani R. Complications of post cochlear implantation in 1027 adults and children. Ann Saudi Med 2019; 39(2): 77-81. 